\title{
INFLUÊNCIA DE FATORES AMBIENTAIS NAS PROPRIEDADES MECÂNICAS DE BIOCOMPÓSITOS DE PLA REFORÇADO COM FIBRA DE COCO E BORRA DE CAFÉ
}

\author{
Erika Bomfim dos Santos ${ }^{1}$, Fábio Roberto Passador ${ }^{1}$, Larissa Stieven Montagna ${ }^{1 *}$ \\ ${ }^{1}$ Laboratório de Tecnologia de Polímeros e Biopolímeros (TecPBio), Universidade Federal de São Paulo, Rua Talim 330, CEP \\ 12231-280, São José dos Campos, São Paulo, Brasil.
}

*E-mail: larissa.s.montagna@gmail.com

\section{RESUMO}

O desenvolvimento de biocompósitos poliméricos tem despertado tanto o interesse científico quanto industrial em desenvolver produtos ecologicamente corretos para diversas aplicações. Desta forma, este trabalho de pesquisa teve o intuito de desenvolver e caracterizar compósitos biodegradáveis de poli (ácido lático) (PLA), reforçado com diferentes porcentagens (5\% e $15 \% \mathrm{~m} / \mathrm{m}$ ) de fibra de coco (FC) e borra de café (BC), sem tratamento superficial. Tanto a FC quanto a BC são popularmente tratadas como resíduos sólidos e apresentam grande potencial para serem utilizados como cargas naturais agindo como reforço e também como aceleradores da biodegradação em matrizes poliméricas para a obtenção de um material biodegradável. Os compósitos foram processados em um homogeneizador DRAIS seguido de prensagem a quente. As amostras foram caracterizadas pela análise visual (imagens macroscópicas) e medidas de ângulo de contato (goniômetro), bem como foram submetidas aos testes de absorção de água e envelhecimento em estufa, seguido do ensaio de resistência ao impacto Izod. Após, verificou-se absorção de água, perda de massa, análise visual, resistência mecânica ao impacto Izod e a caracterização morfológica por microscopia eletrônica de varredura (MEV) da superfície de fratura das amostras após ensaio mecânico. A partir dos resultados obtidos verificou-se a boa adesão entre as cargas naturais e a matriz de PLA, resultando em valores de resistência ao impacto próximo ao do PLA puro, porém os fatores ambientais influenciaram diretamente nessas propriedades mecânicas, tendo a umidade interferida na redução e no envelhecimento do leve acréscimo dos valores de resistência ao impacto.

Palavras-chave: Poli(ácido lático). Fibra de Coco. Borra de Café. Compósitos. Polímeros Biodegradáveis.

\section{Introdução}

Os polímeros biodegradáveis vêm se destacando pelo potencial de substituir, em determinadas aplicações, os polímeros sintéticos e convencionais, derivados de fontes não renováveis, que são descartados incorretamente no meio ambiente, acarretando impactos ambientais e à saúde da população [1-2]. Dentre as inúmeras vantagens apresentadas pelos polímeros biodegradáveis existem algumas limitações no seu processamento, uma vez que degradam em altas temperaturas, e consequentemente interferem na sua aplicação como produto final, comparado aos polímeros convencionais [3-4].

O poli(ácido lático) - PLA é um poliéster, termoplástico, que pode ser semicristalino ou amorfo, e sintetizado a partir do ácido lático proveniente de fontes renováveis como a cana de açúcar, o milho e o trigo. Além disso, o PLA apresenta boas propriedades mecânicas como elevada rigidez, resistência mecânica e alto módulo de elasticidade, quando comparado às poliolefinas como o polietileno (PE), além de possuir a vantagem de ser biodegradável, ampliando a sua gama de aplicações [5-6].

Desta forma, cria-se a necessidade de pesquisar maneiras de melhorar as propriedades dos polímeros biodegradáveis, sendo uma das alternativas o desenvolvimento de compósitos biodegradáveis, por meio da combinação de dois ou mais materiais na forma bifásica, sendo a fase contínua correspondente à matriz polimérica biodegradável, e a fase descontinua constituída pela carga e/ou reforço [7-8].

A incorporação de reforços de origem natural em matriz polimérica é uma alternativa sustentável, pois reduz a quantidade de material polimérico utilizado na fabricação do produto aliado à diminuição de resíduos descartados de forma incorreta no meio ambiente. Esses biocompósitos podem ser compostados ao final da sua utilização, além de acrescentar propriedades com melhor custo benefício ao produto final [9-10]. 
A borra de café e fibra de coco, são classificados como resíduos sólidos possuem pouco valor agregado e fácil acesso, são boas opções a serem utilizadas no desenvolvimento de biocompósitos, não somente pelas possíveis melhorias que ambas podem proporcionar ao material, mas também visando a diminuição desses resíduos amplamente gerados pela população.

A fibra de coco é uma fibra lignocelulósica nativa brasileira fruto do coqueiro (cocos nucifera). A produção do coco no âmbito nacional é direcionada para o consumo da sua água e polpa, gerando subprodutos sólidos que em suma representa aproximadamente $85 \%$ de sua massa total [11]. Desta forma obtêm-se cerca de $10 \mathrm{~kg}$ de fibras a partir de 10.000 unidades de coco, e sua degradação demora em torno de 8 anos, causando um grande volume em aterros sanitários e lixões [12].

Desta forma, diversas pesquisas estão sendo realizadas [1314] com o intuito de melhorar as propriedades físicas, mecânicas e térmicas de matrizes poliméricas biodegradáveis com a incorporação de fibras naturais de origem vegetal, uma vez que a variedade de plantas disponíveis na biodiversidade do nosso planeta, além de ser um recurso renovável e são menos abrasivas que as fibras artificiais usualmente utilizadas como reforços, como a fibra de vidro e de carbono [15-16].

Mulinare e colaboradores [15] incorporaram 10\% em massa de fibra de coco tratadas quimicamente (tratamento alcalino) na matriz de resina poliéster. Os autores verificaram que o tratamento superficial das fibras não influenciou nos resultados de tração e de fadiga na matriz polimérica.

Por outro lado, de acordo com as informações da Empresa Brasileira de Pesquisa Agropecuária (EMBRAPA), o consumo interno de café no Brasil é cerca de 1,07 milhões de toneladas, além disso, a perspectiva do consumo de café em pó para os próximos anos é de um aumento de 3,3\% [17-18]. Assim sendo, é imensurável a quantidade de pó de café pós-uso, borra de café, descartado por residências e comércios no planeta, gerando aumento no volume desses resíduos que são depositados no meio ambiente, ou queimados nas caldeiras para geração de vapor ou destinados para a agricultura para serem utilizados como adubo orgânico [19-20]. A borra de café apresenta em sua composição lipídios, proteínas e fibras [21], logo apresenta características estruturais de uma fibra natural, porém por ser um resíduo não apresenta aplicação na indústria [22-23].

Sendo assim, uma maneira de minimizar essa problemática, aliada à destinação nobre para esse resíduo, é o reaproveitamento de borra de café, por meio da sua utilização como carga e/ou reforço natural em compósitos poliméricos [22-24]. Existe um número limitado na literatura de trabalhos relacionados com o desenvolvimento de compósitos poliméricos utilizando a borra de café como carga e/ou reforço [25-26].

O estudo desenvolvido por Oliveira [22] visou o potencial uso como reforço da borra de café no desenvolvimento de compósitos biodegradáveis utilizando a matriz poli( $\varepsilon$ caprolactona)-PCL. A autora modificou quimicamente a borra do café por mercerização seguida da acetilação e posteriormente por silanização, com o intuito de melhorar a interface da borra de café com a matriz de PCL. Nessa pesquisa, a autora adicionou concentrações de 5\%,10\% e 15\% em massa da carga (natural e quimicamente tratada) na matriz de PCL, por alto cisalhamento seguido de fusão, e após, realizou ensaio de biodegradação em solo por 120 dias dos compósitos desenvolvidos. Como resultado final, verificou-se que o tratamento químico e a adição de borra de café na matriz de PCL aceleraram a biodegradação em solo dos compósitos desenvolvidos. Com relação à resistência à tração, a presença da borra de café não alterou as propriedades mecânicas dos compósitos ao comprar com a matriz de PCL puro.

Essabir e colaboradores [25] desenvolveram biocompósitos de polipropileno (PP) com diferentes teores de borra de café (5, 10,15 e $20 \%$ em massa) utilizando agentes compatibilizantes (silano e estireno-etileno-buteno-estireno-enxertado com anidrido maleico), processados por extrusão seguidos de moldagem por injeção. Os autores analisaram a influência da borra de café nas propriedades térmicas, reológicas e mecânica. Os resultados indicaram boa dispersão, distribuição e melhor interação interfacial da borra de café na matriz de PP utilizando os agentes compatibilizantes, além de leve aumento das propriedades mecânicas. Resultados semelhantes foram observados por Wu [26], que caracterizou e estudou a biodegradação de compósitos de PLA/borra de café enxertado com anidrido maleico. O autor verificou melhora na dispersão da borra de café na matriz de PLA com a presença do agente compatibilizante, porém foi observada redução da temperatura de fusão desses compósitos, na qual resultou na diminuição da temperatura de processamento. Além do mais, a presença do agente compatibilizante reduziu o tempo de biodegradação dos compósitos de PLA/borra de café.

As fibras e cargas naturais apresentam características hidrofílicas e os materiais poliméricos são hidrofóbicos, sendo essa incompatibilidade capaz de interferir negativamente na adesão entre carga-matriz polimérica, resultando na redução e/ou perdas nas propriedades finais do compósito desenvolvido [8]. Por conseguinte, inúmeros tratamentos superficiais nas fibras podem ser realizados com o intuito de melhorar a interface entre carga-polímero [27-28], porém, geralmente, esses tratamentos utilizam solventes e reagentes químicos, que acabam acarretando na geração de poluentes no meio ambiente, e muitas vezes esses tratamentos superficiais nas fibras não resultam em resultados 
satisfatórios e aumentos significativos nas propriedades térmicas, mecânicas e físicas dos compósitos [29].

Portanto, o presente trabalho visou à utilização de materiais naturais, a fibra de coco e a borra de café, conforme recebido, sem nenhum tratamento preliminar, visto que, com base nos estudos existentes, a presença de agente compatibilizante e/ou o tratamento químico das cargas naturais, não influenciam de forma significativa na melhoria da dispersão dessas cargas na matriz polimérica e tampouco aumentam de forma significativa as propriedades mecânicas gerais. Além do mais, este trabalho visou ao não uso de nenhum produto químico que pudesse interferir e prejudicar o meio ambiente. Sendo assim, este estudou a introdução das cargas naturais (fibra de coco e borra de café in natura) na matriz biodegradável de poli(ácido lático), PLA, um polímero biodegradável de fonte renovável, a fim de verificar o comportamento dessas cargas na matriz de PLA, além da exposição desses compósitos desenvolvimentos ao ensaio de absorção de água e ao envelhecimento em estufa, e análise das características morfológicas e mecânicas, com o intuito de desenvolver um material ecologicamente sustentável.

\section{Parte Experimental ou Metodologia}

\subsection{Materiais}

A matriz polimérica utilizada foi o poli(ácido lático) (PLA), fornecido pela Biopolymers (P7051). A fibra de coco (FC) utilizada foi fornecida pela empresa Coco Verde Reciclado (Rio de Janeiro/RJ). A fibra de coco foi cortada manualmente ( 2 $\mathrm{cm})$, como pode ser visto pela Figura 1-A. Antes do processamento, a FC foi seca em estufa com circulação de ar a $60{ }^{\circ} \mathrm{C}$ durante 24 horas. A borra de café (BC) foi coletada do refeitório do Instituto de Ciência e Tecnologia da Universidade Federal de São Paulo no campus de São José dos Campos (ICTUNIFESP/SJC) (Fig. 1-B). Após coletada, a borra de café foi seca em estufa com circulação de ar a $100{ }^{\circ} \mathrm{C}$ por 72 horas, e também foi seca imediatamente antes do uso no processamento por 24 horas a $60^{\circ} \mathrm{C}$.

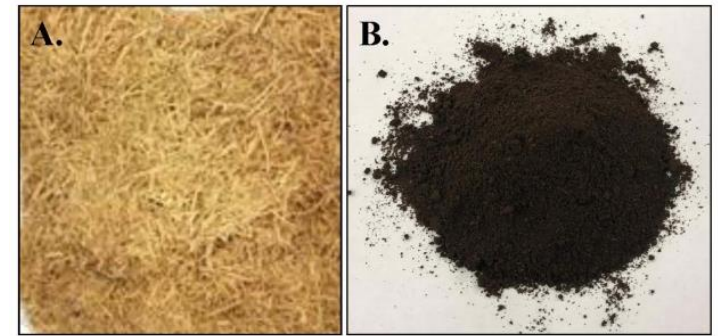

Figura 1 - Cargas utilizadas: fibra de coco (A) e borra de café (B).

\subsection{Métodos}

2.2.1. Preparação das amostras de PLA puro e dos compósitos de PLA/FC, PLA/BC e PLA/BC/FC

Os compósitos biodegradáveis de PLA com diferentes porcentagens de fibra de coco e borra de café $(0 ; 5 \%$ e $15 \%$ em massa) foram preparados por meio de mistura em um misturador termocinético rotacional (DRAIS, modelo $\mathrm{MH} 50 \mathrm{H}$ da $\mathrm{MH}$ Equipamentos) em que a mistura dos materiais e a fusão do polímero ocorrem em decorrência do atrito e rotação (3000 rpm). Os materiais foram colocados na câmara de mistura e após 1 minuto ocorreu à fusão/amolecimento do PLA e mistura com os demais constituintes. Após homogeneização, as amostras foram prensadas a quente utilizando uma prensa hidropneumática $(\mathrm{MH}$ Equipamentos, modelo PR8H) a $190{ }^{\circ} \mathrm{C}$, com tempo de prensagem de 3 min e pressão de 5 bar. A Tabela 1 apresenta as composições dos biocompósitos biodegradáveis de PLA puro e dos biocompósitos com diferentes porcentagens de FC e BC (5\% e $15 \% \mathrm{~m} / \mathrm{m})$.

Tabela 1 - Porcentagens e composições das amostras.

\begin{tabular}{cccc}
\hline AMOSTRAS & PLA & $\begin{array}{c}\text { COMPOSIÇÕES (\% EM MASSA) } \\
\text { FIBRA DE } \\
\text { COCO }\end{array}$ & $\begin{array}{c}\text { BORRA DE } \\
\text { CAFE }\end{array}$ \\
\hline PLA puro & 100 & 0,0 & 0,0 \\
PLA/5\% FC/BC & 95 & 2,5 & 2,5 \\
PLA/15\% FC/BC & 85 & 7,5 & 7,5 \\
PLA/5\% FC & 95 & 5,0 & 0,0 \\
PLA/15\% FC & 85 & 15,0 & 0,0 \\
PLA/5\% BC & 95 & 0,0 & 5,0 \\
PLA/15\% BC & 85 & 0,0 & 15,0 \\
\hline
\end{tabular}

\subsubsection{Caracterização de superfície do PLA puro e dos compósitos}

A determinação do ângulo de contato foi realizada por meio de um goniômetro (KRÜSS - DSC 100, Drop Shape Analyzer). O líquido utilizado foi água destilada pelo método da gota séssil $\left(1.0 \mu \mathrm{L}, 25^{\circ} \mathrm{C}\right)$. Foram realizadas 5 medidas em cada amostra a fim de avaliar o caráter superficial das amostras de PLA puro e dos compósitos (hidrofílico ou hidrofóbico).

\subsubsection{Absorção de água}

As amostras de PLA puro e dos compósitos foram submersos em um banho contendo água destilada (Nova Instruments) a 60 ${ }^{\circ} \mathrm{C}$ por 7 dias. Ao término do ensaio, as amostras foram secas com papel toalha. O cálculo da taxa de absorção de água das amostras foi realizado de acordo com a Equação 1, onde $M_{f}$ é a 
massa final e $M_{i}$ é a Massa inicial. O ensaio foi realizado em triplicata.

$$
\text { Absorção de água }(\%)=\frac{(M f . M i)}{M i} \times 100
$$

\subsubsection{Envelhecimento na estufa}

As amostras de PLA puro e dos compósitos foram expostas em estufa com circulação de ar (Sterilifer, Modelo: SXCR 40) à $60{ }^{\circ} \mathrm{C}$ por 30 dias. $\mathrm{O}$ cálculo da perda de massa das amostras foi realizado de acordo com a Equação 2, onde $M_{f}$ é a massa final e $M_{i}$ é a massa inicial. O ensaio foi realizado em triplicata.

$$
\text { Perda de massa }(\%)=\frac{(M f . M i)}{M i} \times 100
$$

\subsubsection{Análise visual}

As amostras foram analisadas visualmente por meio de imagens fotográficas realizadas antes e após o ensaio de absorção de água e de envelhecimento em estufa, com o intuito de verificar as modificações macroscópicas após os ensaios de envelhecimento realizados.

\subsubsection{Caracterização mecânica por resistência ao impacto Izod}

A resistência ao impacto Izod das amostras foi realizada de acordo com a norma ASTM D256, utilizando um impactor da CEAST/Instron modelo 9050, e martelo de 1,0 J. Foram analisados 5 corpos de prova de cada amostra.

\subsubsection{Características morfológicas}

A análise morfológica da superfície de fratura das amostras, de PLA puro, PLA/5\% BC, PLA/15\% BC, PLA/5\% FC, PLA $/ 15 \%$ FC, PLA/2,5\% BC/2,5\% FC e PLA/7,5\% BC/7,5\% FC foi obtida após o ensaio mecânico de impacto, e foi analisado em um microscópio eletrônico de varredura (MEV) da marca Inspect S50 - FEI Company ${ }^{\circledR}$, operado a $10 \mathrm{keV}$. As amostras foram criofraturadas com nitrogênio líquido, e fixadas em stubs de alumínio e metalizadas com ouro.

\section{Resultados e discussões}

A Tabela 2 apresenta os valores de ângulo de contato das amostras de PLA puro e dos biocompósitos (PLA/5\% BC, PLA/15\% BC, PLA/5\% FC, PLA/15\% FC, PLA/5\% BC/FC e $\mathrm{PLA} / 15 \% \mathrm{BC} / \mathrm{FC})$. O ângulo de contato permite verificar a afinidade do líquido, a água neste caso, com o material. Desta forma, o comportamento está relacionado com as tensões e interações superficiais, ou seja, à capacidade de molhamento de acordo com o ângulo de contato que pode ser classificado como superfícies hidrofílicas $\left(\theta<90^{\circ}\right)$, hidrofóbicas $\left(90^{\circ}<\theta<150^{\circ}\right)$ ou totalmente hidrofóbicas $\left(\theta>150^{\circ}\right)$. O PLA é um polímero apolar de baixa energia de superfície, o que dificulta sua molhabilidade, já as fibras naturais são hidrofílicas, isto é, são fibras lignocelulósicas, que possuem o caráter de absorver umidade.

Devido a essa diferença de hidrofilicidade entre as cargas naturais e a matriz polimérica, muitos pesquisadores optam por realizar algum tipo de tratamento superficial nas cargas, com o intuito de melhorar a afinidade e a adesão entre a carga e a matriz polimérica.

Observou-se uma redução do ângulo de contato com o aumento da quantidade de carga, independente do tipo, em relação ao PLA puro, um comportamento já esperado. Esta redução deve-se, provavelmente, à baixa afinidade entre matriz $\mathrm{e}$ reforço. Os resultados similares entre o tipo de carga, ou mesmo considerando-se a mistura de cargas, estão relacionados à natureza semelhante das cargas. Portanto, todos os compósitos desenvolvidos apresentaram um comportamento hidrofílico, ou seja, possuem boa interação com a água [30-31].

As fibras naturais apresentam comportamento hidrofílico, devido à presença dos grupos hidroxila que apresentam em sua estrutura, desta forma absorvem água da atmosfera. Esse comportamento hidrofílico das fibras pode ser uma problemática, visto que a absorção de água pode acarretar em problemas como o surgimento de trincas na matriz polimérica, instabilidade dimensional do produto gerado além da redução das propriedades mecânicas [32]. Por outro lado, esse comportamento hidrofílico apresentado pelos compósitos desenvolvidos poderá auxiliar na degradação por hidrólise, visto que esse tipo de degradação é mais susceptível para os poliésteres, devido à presença do grupo -COO- em suas estruturas, sendo assim, auxilia na aceleração de biodegradação desses materiais após o seu uso e descarte em ambientes úmidos [33-34]. 
Tabela 2 - Valores e imagens dos ângulos de contato das amostras de PLA puro e dos compósitos.

\begin{tabular}{|c|c|c|}
\hline AMOSTRAS & $\begin{array}{l}\hat{A} \text { NGULO DE } \\
\text { CONTATO ( }\left(^{\circ}\right)\end{array}$ & IMAGEM \\
\hline PLA puro & 98 & \\
\hline $\mathrm{PLA} / 5 \% \mathrm{FC}$ & 72 & \\
\hline PLA/15\% FC & 51 & \\
\hline PLA/5\% BC & 65 & \\
\hline PLA/15\% BC & 56 & \\
\hline $\mathrm{PLA} / 5 \% \mathrm{FC} / \mathrm{BC}$ & 67 & \\
\hline PLA/15\% FC/BC & 58 & \\
\hline
\end{tabular}

Os resultados da absorção de água no período de sete dias estão apresentados na Figura 2, no qual é possível observar os valores consistentes com o teor de carga incorporada aos biocompósitos desenvolvidos (5\% e 15\% em massa). É possível observar, que ambos os compósitos de mesmas composições, absorveram quantidades semelhantes de água, ou seja, apresentaram valores de absorção próximos e coerentes com a quantidade de carga presente nos biocompósitos [34-35]. Desta forma, ao comparar a amostra de PLA puro com os biocompósitos, observou-se menor porcentagem de absorção, o que já era esperado, pois as cargas naturais absorvem umidade, pois suas estruturas possuem grupos hidroxila, que possuem os principais constituintes das fibras naturais lignocelulósicas, que interagem com água por meio de ligações de hidrogênio [36-37]. Sendo assim, os valores de absorção de água estão de acordo com os valores de ângulo de contato.

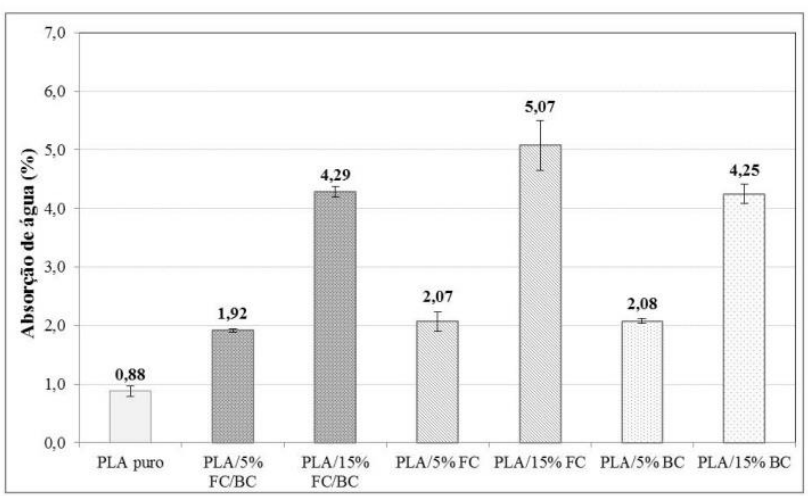

Figura 2 - Porcentagem de absorção de água das amostras de PLA puro e dos compósitos.

A Figura 3 apresenta os valores de porcentagem de perda de massa das amostras de PLA puro e dos biocompósitos após de 30 dias de exposição em estufa à temperatura de $60{ }^{\circ} \mathrm{C}$. Ao analisar os resultados, observou-se que os compósitos apresentaram aumento na porcentagem perda de massa com o aumento do teor de carga natural adicionada a matriz polimérica. Este fato pode estar relacionado com a natureza das cargas utilizadas, que podem ser mais susceptíveis a degradação térmica comparada ao PLA puro. Desta forma, a adição de cargas naturais influenciou na degradação térmica do compósito. Porém, o aspecto das amostras não modificou consideravelmente, como pode-se observar na análise visual, apresentada a seguir na Figura 4. 


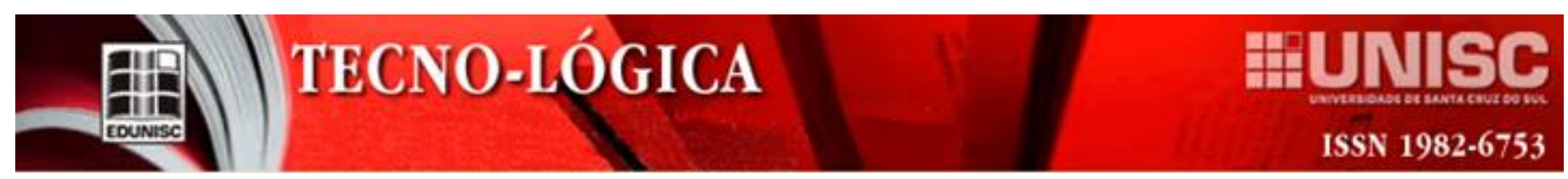

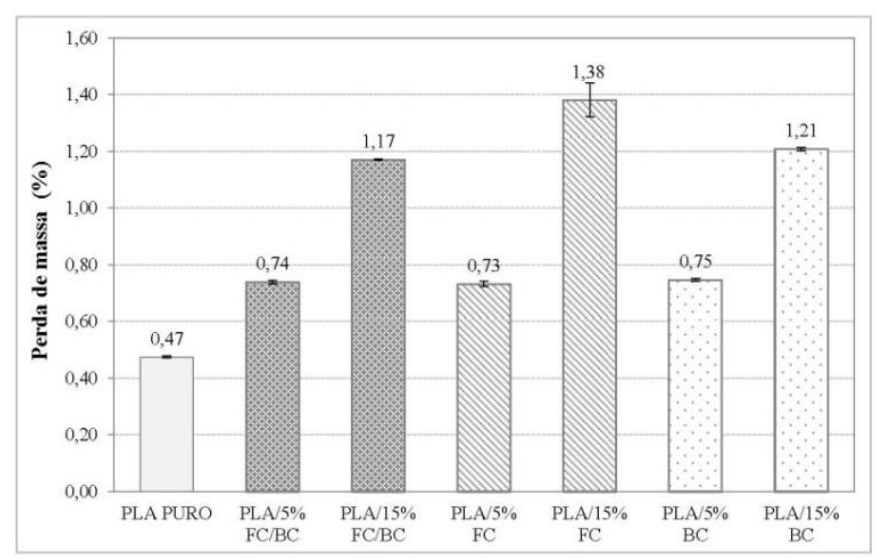

Figura 3- Porcentagem de perda de massa das amostras de PLA puro e dos compósitos após 30 dias de exposição na estufa.

A Figura 4 apresenta a análise visual das amostras, PLA puro e dos compósitos, no tempo inicial (Fig. 4-A), após ensaio de absorção de água durante 7 dias (Fig.4-B) e após ensaio de envelhecimento em estufa à $60{ }^{\circ} \mathrm{C}$ por 30 dias (Fig. 4-C). Observa-se que após o ensaio de absorção de água por 7 dias as amostras apresentaram mudanças significativas na coloração, ficaram mais opacas e sem distinção das cores ao serem comparadas com as amostras no tempo inicial. $\mathrm{O}$ ambiente em que as amostras ficaram expostas, ou seja, a combinação de umidade e aquecimento $\left(60{ }^{\circ} \mathrm{C}\right)$ pode ter influenciado na modificação das amostras. Já por meio da análise visual das amostras após envelhecimento em estufa notou-se que não ocorreram mudanças significativas ao comparar com as amostras no tempo inicial, sendo assim, pode-se concluir que as amostras mostraram ser mais resistentes à degradação térmica do que à ação da água.

A Figura 5 apresenta os resultados de resistência ao impacto Izod das amostras de PLA puro e dos compósitos (PLA/5\% BC, PLA/15\% BC, PLA/5\% FC, PLA $/ 15 \%$ FC, $\mathrm{PLA} / 5 \% \mathrm{BC} / \mathrm{FC}$ e PLA/15\% BC/FC) no tempo inicial, após ensaio de absorção de água por sete dias e após envelhecimento em estufa por 30 dias. Observou-se nas amostras no tempo inicial, que a incorporação das cargas naturais, fibra de coco e borra de café, na matriz de PLA não apresentou nenhuma tendência ao aumento à resistência ao impacto Izod; ou seja, não ocorreu mudança significativa nos valores de resistência ao impacto dos biocompósitos desenvolvidos, apresentando resultados bem próximos ao PLA puro, dentro do desvio padrão.

Entretanto é mais evidente observar que nas amostras contendo a fibra de coco, PLA/5\% FC e PLA/15\% FC, verificouse redução de $7,6 \%$ e $13,6 \%$ na resistência ao impacto, respectivamente, em comparação com o PLA puro. Este comportamento pode ser devido à fraca adesão da matriz de PLA com a fibra de coco, ou seja, não resultou numa boa transferência de carga da matriz para a fibra. Resultado semelhante foi observado por Jesus [38], que estudou a influência de tratamentos superficiais em fibras de sisal e em fibras de coco incorporadas em matriz de poliéster de polihidroxibutirato PHB. Contudo, após analisar os resultados de resistência ao impacto, o autor notou que o tratamento superficial nas fibras de coco foi desnecessário, pois não ocorreu mudança significativa ao comparar com a matriz de PHB puro.

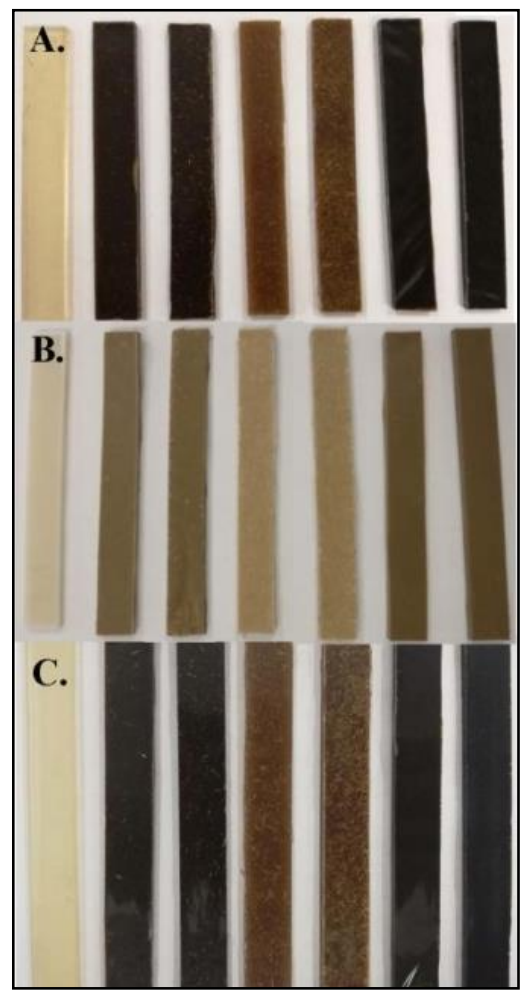

Figura 4 - Análise visual das amostras de PLA puro e dos compósitos (PLA/5\% BC, PLA $/ 15 \%$ BC, PLA $/ 5 \%$ FC, PLA/15\% FC, PLA/5\% BC/FC e PLA/15\% $\mathrm{BC} / \mathrm{FC}$ ): no tempo inicial (A), após ensaio de absorção de água por 7dias (B) e após envelhecimento em estufa por 30 dias (C).

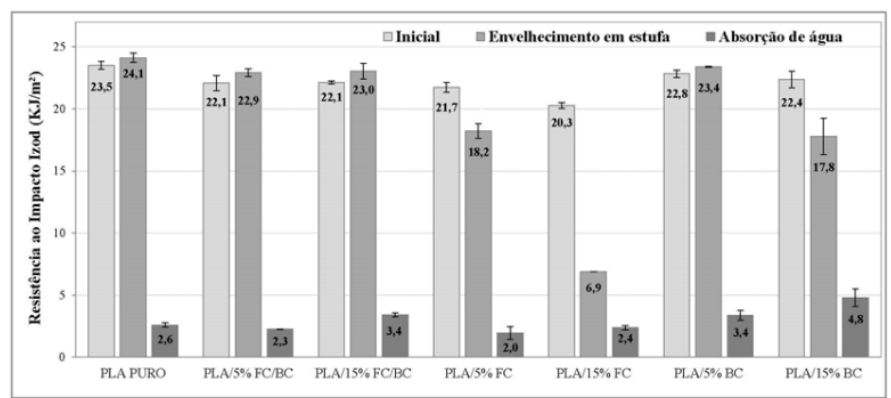

Figura 5 - Resistência ao impacto Izod do PLA puro e dos compósitos (PLA/5\% BC, PLA/15\% BC, PLA/5\% FC, PLA/15\% FC, PLA/5\% BC/FC e PLA/15\% $\mathrm{BC} / \mathrm{FC}$ ) antes e após o ensaio de absorção de água e após envelhecimento em estufa. 
As amostras submetidas ao ensaio de envelhecimento em estufa a $60^{\circ} \mathrm{C}$ por 30 dias apresentaram mudanças nos valores de resistência ao impacto. As amostras de PLA puro, os biocompósitos com 5\% e 15\% em massa de FC/BC (PLA/5\% FC/BC e PLA/15\% FC/BC) e a amostra de PLA/5\% BC apresentaram aumento de 2,5\%, 3,6\%, $4 \%$ e 2,6\%, respectivamente, ao comparar com as suas composições no tempo inicial. Este fato pode estar relacionado com a influência da remoção de umidade das cargas naturais durante $o$ envelhecimento em estufa, favorecendo ao aumento da resistência ao impacto Izod. Vale ressaltar ainda que o elevado tempo de exposição em altas temperaturas pode acarretar em mudanças estruturais, como por exemplo, aumento do alinhamento das cadeias e do grau de cristalinidade da matriz polimérica [39].

Já as amostras submetidas ao ensaio de absorção de água apresentam elevada redução nos valores de resistência ao impacto, pois devido ao período em que as amostras ficaram submersas em água, absorveram umidade, conforme observado na Figura 2. A água pode migrar para a interface entre a fibra e a matriz, atuando como concentrador de tensão, fragilizando a amostra. Além disso, devido à baixa adesão entre matriz-carga natural e o caráter hidrofílico das cargas, facilitou a absorção de umidade, influenciando diretamente nas propriedades mecânicas das amostras, pois a umidade pode ter feito com que as amostras degradassem por hidrolise, tornando o material mais quebradiço, resultando na diminuição da resistência ao impacto [40-41].

A Figura 6 apresenta a morfologia da superfície após ensaio mecânico de impacto das amostras de PLA puro e dos biocompósitos desenvolvidos (PLA/5\% BC, PLA/15\% BC, PLA $/ 5 \%$ FC, PLA $/ 15 \%$ FC, PLA $/ 5 \%$ BC/FC e PLA/15\% BC/FC) com ampliação de 500x. A Figura 6A apresenta uma morfologia homogênea e lisa, referente ao PLA puro. Observa-se nas superfícies de fraturas dos biocompósitos contendo a menor quantidade de cargas (FC e BC), 5\% em massa, (Figura 6BDF) apresentaram uma morfologia homogênea, no qual as cargas de BC e FC apresentaram-se bem dispersas na matriz de PLA, ou seja, ocorreu boa interação e adesão entre as cargas com a matriz polimérica de PLA.

As micrografias da Figura 6-CEG são referentes aos biocompósitos de PLA contendo 15\% em massa das cargas (BC e FC), nas quais é possível observar a separação de fases, onde as cargas (BC e FC) encontram-se dispersas na matriz de PLA. Também é possível perceber a presença de vazios na fase dispersa que pode ser devido à remoção das cargas de BC e FC no ensaio mecânico de impacto. Sendo assim, notou-se que o maior teor das cargas de $\mathrm{BC}$ e FC e a combinação de ambas (15\% em massa) influenciaram negativamente na adesão entre as fases das cargas utilizas e da matriz de PLA. Porém observou-se boa adesão interfacial da BC na matriz de PLA, tanto com 5\% e $15 \%$ de carga (Figura 6BC). Esse comportamento está de acordo com os resultados de resistência ao impacto Izod apresentado na Figura 5.
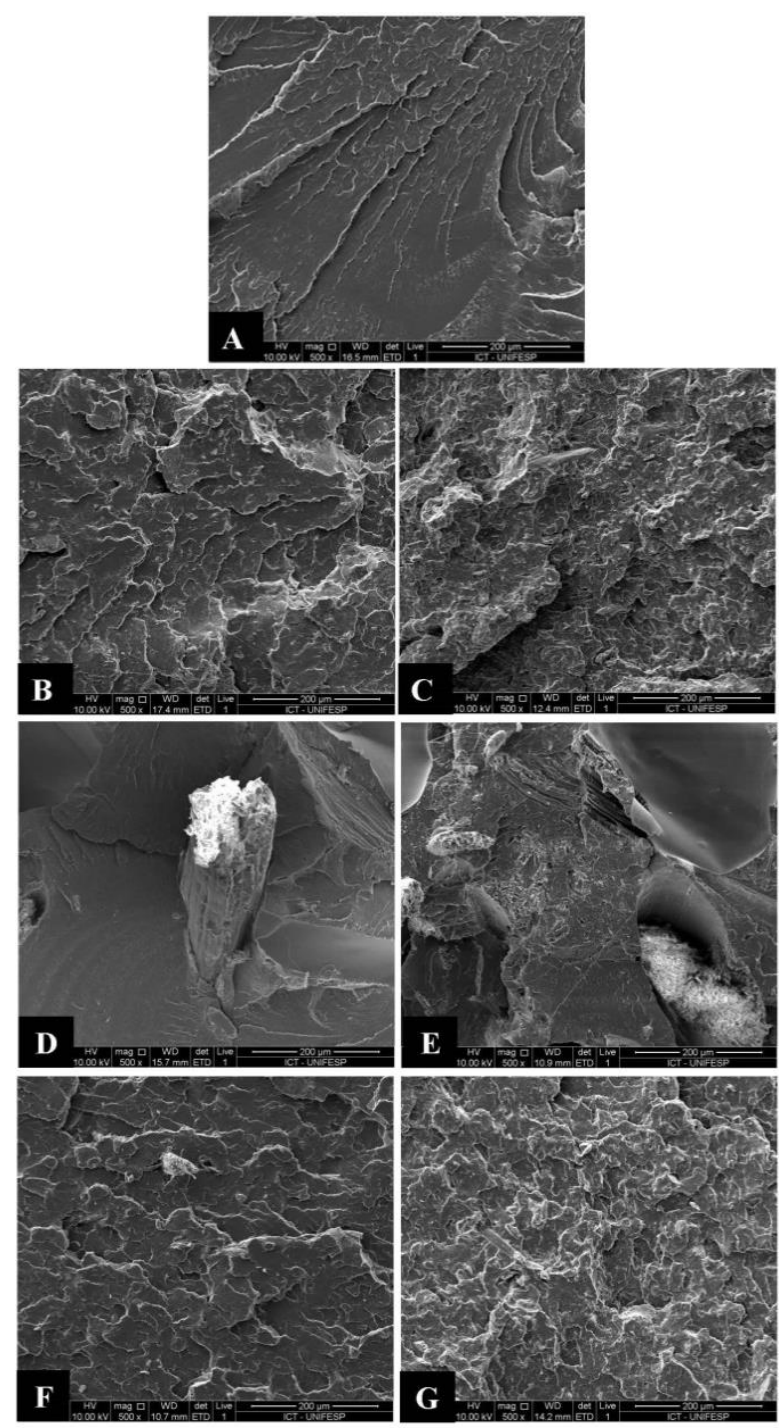

Figura 6 - Micrografias de MEV da superfície de fratura após ensaio mecânica das amostras: PLA puro (A), PLA/5\% BC (B), PLA/15\% BC (C), PLA/5\% FC (D), PLA/15\% FC (E), PLA/5\% BC/FC (F) e PLA/ $15 \%$ BC/FC (G) com ampliação de 500x. 


\section{Conclusão}

Este trabalho de pesquisa apresentou o desenvolvimento e a caracterização de biocompósitos de PLA contendo diferentes porcentagens $(5 \%$ e $15 \% \mathrm{~m} / \mathrm{m})$ de cargas naturas de fibra de coco e borra de café sem tratamento superficial.

Os valores de ângulo de contato mostraram um comportamento hidrofílico de todas as amostras desenvolvidas. A análise morfológica da superfície de fratura criogênica mostrou boa adesão das cargas naturais com a matriz de PLA.

O ensaio de absorção de água indicou que conforme aumentou o teor de carga na matriz de PLA, aumetou consequentemente a porcentagem de absorção de água nos compósitos desenvolvidos, este resultado já era esperado devido ao comportamento hidrofílico das cargas naturais.

O teste de envelhecimento em estufa indicou que os compósitos foram mais susceptíveis à degradação térmica que a matriz de PLA puro, esse comportamento pode estar relacionado com a natureza das cargas utilizadas, que podem ser mais susceptível a degradação térmica que o PLA puro.

O resultado de resistência ao impacto Izod indicou que a presença das cargas, fibra de coco e borra de café, na matriz de PLA não influenciaram na perda significativa dessa propriedade mecânica estudada. Porém os fatores ambientais, absorção de água e envelhecimento em estuda, aos quais as amostras foram submetidas interferiram nos valores de resistência ao impacto. A umidade influenciou drasticamente na degradação hidrolítica das amostras, resultando em redução da resistência ao impacto. Entretanto o envelhecimento na estufa resultou em leve aumento dos valores de resistência ao impacto.

\section{Agradecimentos}

Os autores agradecem à empresa Coco Verde/RJ pelo fornecimento das fibras de coco e ao Instituto Tecnológico de Aeronáutica (ITA) pelas medidas de ângulo de contato.

Apoio Financeiro: FAPESP (2014/04900-9 e 2016/199789) e CNPq (442081/2014-6 e 405675/2018-6).

\section{INFLUENCE OF ENVIRONMENTAL FACTORS ON THE MECHANICAL PROPERTIES OF PLA BIOCOMPOSITES REINFORCED WITH COCONUT FIBER AND COFFEE GROUNDS}

ABSTRACT: The development of polymeric biocomposites has aroused both scientific and industrial interest in developing ecologically correct products for various applications. In this way, this work aimed to develop and characterize biodegradable by poly(lactic acid) composites (PLA), reinforced with different percentages (5\% and $15 \mathrm{wt} \%)$ of coconut fiber (CF) and coffee grounds (CG) without surface treatment. Both $\mathrm{CF}$ and $\mathrm{CG}$ are popularly treated as solid waste and have great potential to be used as natural fillers acting as reinforcement and also as accelerators of biodegradation in polymer matrices, in order to obtain a biodegradable material. The composites were processed into a DRAIS homogenizer followed by hot pressing. Samples were characterized by visual analysis (macroscopic images) and contact angle measurements (goniometer). The samples were subjected to the water absorption test and aging in an oven, followed by Izod impact resistance test. Afterwards, the water absorption, mass loss, visual analysis, Izod impact mechanical strength and the scanning electron microscopy (SEM) morphological characterization of the fracture surface of the samples after mechanical test were verified. From the results obtained, the good adhesion between the natural loads and the PLA matrix was verified resulting in values of impact resistance close to the neat PLA, but the environmental factors directly influenced these mechanical properties, being the humidity interfering in the reduction and the aging in the slight increase of the values of impact resistance.

Keywords: Poly (lactic acid). Coco Fiber. Coffee grounds. Composites. Biodegradable.

\section{Referências}

[1] RYDZ, J.; MUSIOŁ, M.; ZAWIDLAK-WĘGRZYŃSKA, B.; SIKORSKA, W. Chapter. 14 - Present and Future of Biodegradable Polymers for Food Packaging Applications. Biopolymers for Food Design. Handbook of Food Bioengineering, 2018, 431-467.

[2] LUYT, A.S.; MALIK, S. S. 16 - Can Biodegradable Plastics Solve Plastic Solid Waste Accumulation? Plastics to Energy Fuel, Chemicals, and Sustainability Implications. Plastics Design Library, 2019, 403-423.

[3] LAYCOCK, B.; NIKOLI'C, M.; COLWELL, J. M.; GAUTHIER, E.; HALLEY, P.; BOTTLE, S.; GEORGE, G. Lifetime prediction of biodegradable polymers. Progress in Polymer Science, v. 71, p. 144-189, 2017.

[4] ALIASHTER, S. OVERVIEW OF BIODEGRADABLE POLYMERS. INTRODUCTION TO BIOPLASTICS ENGINEERING. PLASTICS DESIGN LIBRARY, P.19-30, 2016.

[5] JIN, F-L.; HU, R-R.; PARK, S-J. Improvement of thermal behaviors of biodegradable poly(lactic acid) polymer: A Review. Composites Part B, v. 164, 1,p. 287-296, 2019. 
[6] MURARIU, M.; DUBOIS, P. PLA COMPOSITES: FROM PRODUCTION TO PROPERTIES. ADVANCED DRUG DELIVERY REVIEWS, V. 107, P.1746, 2016.

[7] RHIM, J-W.; KIM, Y-T. CHAPTER 17 - BIOPOLYMER-BASED COMPOSITE PACKAGING MATERIALS WITH NANOPARTICLES. INNOVATIONS IN FOOD PACKAGING (SECOND EDITION), P. 413-442, 2014.

[8] MR, S.; SIENGCHIN, S.; PARAMESWARANPILLAI, J.; JAWAID, M.; PRUNCU, C. I.; KHAN, A. A Comprehensive Review of Techniques for Natural Fibers as Reinforcement in Composites: Preparation, Processing and Characterization. Carbohydrate Polymers. v. 207, p. 108-121, 2019.

[9] SANJAY, M. R.; MADHU, P.; JAWAID, M.; SENTHAMARAIKANNAN, P.; SENTHIL, S.; PRADEEP, S. Characterization and properties of natural fiber polymer composites: A comprehensive review. Journal of Cleaner Production. v. 172, p. 566-581, 2018.

10 MARINELLI, A. L.; MONTEIRO, M. R.; AMBRÓSIO, J. D.; BRANCIFORTI, M. C.; KOBAYASHI, M.; NOBRE, A. D. Desenvolvimento de Compósitos Poliméricos com Fibras Vegetais Naturais da Biodiversidade: Uma Contribuição para a Sustentabilidade Amazônica. Polímeros: Ciência e Tecnologia. v. 18, p. 92-99, 2008.

[11] MARTINS, A. P.; SILVA, P. L. R.; WATANABE, T.; BORELli, C. MARCICANO, J. P. P.; SANCHES, R. A. O Problema do Pós-consumo do Coco no Brasil: Alternativas e Sustentabilidade. Sustentabilidade em Debate - Brasília, v. 7 , p. $44-57,2016$

[12] BAJPAI, P.K.; SINGH, I.; MADAAN, J. Tribological behavior of natural fiber reinforced PLA composites. Wear. v. 297, p. 829-840, 2013.

[13] LEMOS, A. L.; MARTINS, R. M. DESENVOLVIMENTO E CARACTERIZAÇÃO DE COMPÓSITOS POLIMÉRICOS À BASE DE POLI(ÁCIDO LÁTICO) E FIBRAS NATURAIS. POLÍMEROS. V. 24, P.190197, 2014.

[14] NUTHONG, W.; UAWONGSUWAN, P.; PIVSA-ART, W.; HAMADA, H. Impact Property of Flexible Epoxy Treated Natural Fiber Reinforced PLA Composites. Energy Procedia. v.34, p.839-847, 2013.

[15] MULINARI, D.R., BAPTISTA, C.A.R.P., SOUZA, J. V. C., VOORWALD, H.J.C. Mechanical Properties of Coconut Fibers Reinforced Polyester Composites. Procedia Engineering. v. 10, p. 2074-2079, 2011.

[16], J. Y.; JEONG, T.K.; OH, H. J.; YOUN, J. R.; SONG, Y. S. THERMAL STABILITY AND FLAMMABILITY OF COCONUT FIBER REINFORCED POLY(LACTIC ACID) COMPOSITES. COMPOSITES PART B: ENGINEERING. V, 43, P. 2434-2438, 2012.

[17] FERREIRA, T. L.; SANTOS, J. Brasil consome 21,5 milhões de sacas de café em 2017. ABIC - Associação Brasileira da Indústria de Café, 2017.

[18] McNutt, J.; He, Q. S. Spent coffee grounds: A review on current utilization. Journal of Industrial and Engineering Chemistry. In Press, Corrected Proof, December, 2018.
[19] TUNTIWIWATTANAPUN, N.; TONGCUMPOU, C. SEQUENTIAL extraction and reactive extraction processing of spent coffee grounds: An alternative approach for pretreatment of biodiesel feedstocks and biodiesel production. Industrial Crops and Products. v. 117, p. 359-365, 2018.

[20] KOOKOS, I. K. Technoeconomic and environmental assessment of a process for biodiesel production from spent coffee grounds (SCGs). Resources, Conservation and Recycling. v. 134, p.156-164, 2018.

[21] AZEVEDO, A. S. B. Caracterização e Aplicação de fibra de borra de café modificada por tratamento com Peróxido de Hidrogênio alcalino. 2007. 58f. Dissertação (Mestrado em Ciência dos Alimentos) - Universidade Estadual de Londrina, Londrina/PR.

[22] NATÁLIA R. B. O. Caracterização de um compósito polimérico biodegradável utilizando poli(E-caprolactona) e borra de café. 2016. $117 \mathrm{f}$. Dissertação (Mestrado em Engenharia Química) - Faculdade de Engenharia Química da Universidade Estadual de Campinas, UNICAMP, Campinas/SP.

[23] KARMEE, S. K. A spent coffee grounds based biorefinery for the production of bi fuels, biopolymers, antioxidants and biocomposites. Waste Management. V. 72, P. 240-254, 2018.

[24] MEI, L. H.; OLIVEIRA, N. Caracterização de um compósito polimérico biodegradável utilizando Poli ( $\varepsilon$-caprolactona) e borra de café. Polímeros. v. 27, p. 99-109, 2017.

[25] ESSABIR, H.; RAJI, M.; LAAZIZ, S. A.; RODRIQUE, D.; BOUHFID, R.; QAIS, A. E. K. Thermo-mechanical performances of polypropylene biocomposites based on untreated, treated and compatibilized spent coffee grounds. Composites Part B: Engineering. V. 149, P. 1-11, 2018.

[26] WU, C-S. Renewable resource-based green composites of surface-treated spent coffee grounds and polylactide: Characterisation and biodegradability. Polymer Degradation and Stability. v. 121, P. 51-59, 2015.

[27] SOOD, M.; DWIVEDI, G. Effect of fiber treatment on flexural properties of natural fiber reinforced composites: A review. Egyptian Journal of Petroleum. V. 27, Issue 4,p. 775-783, 2018.

[28] FERREIRA, D. P.; CRUZ, J.; FANGUEIRO, R. Chapter 1 - Surface modification of natural fibers in polymer composites. Green Composites for Automotive Applications. Woodhead Publishing Series in Composites Science and Engineering. P. 3-41, 2019.

[29] J SINGH, J. I. P.;DHAWAN, V.; SINGH, S.; JANGID, K. Study of Effect of Surface Treatment on Mechanical Properties of Natural Fiber Reinforced Composites. Materials Today: Proceedings. v. 4, p. 2793-2799, 2017.

[30] SAMPAIO, J. A. Caracterização de Fibras de Celulose Recoberta por Plasma. 2013, 50f. Tese (Tese em Engenharia) - Escola Politécnica, Universidade Federal do Rio de Janeiro, Rio de Janeiro/RJ.

[31] RIBEIRO, Z. A. Ângulo de Contato Formado Por Gota de Água em Superfície de Genótipo de Eucalipito: Um Indicativo de Resistência ao Psilídeode-Concha. Conbraf - Congresso Brasileiro de Fitossanidade, UNESP, Jaboticabal, 2013.

[32] DHAKAL, H.N.; ZHANG, Z.Y.; RICHARDSON, M. O. Effect of water absorption on the mechanical properties of hemp fibre reinforced unsaturated polyester composites. Composites Science Technology. vol. 67: p. 1674-1683, 2007.

[33] DERVAL, S. R.; FILHO, R. P. Biodegradação: um ensaio com polímeros. Editora Universitária São Francisco e editora Moara, 2003.

[34] HALIP, J.A.; HUA, L. S.; ASHAARI, Z.; TAHIR, P. M.; CHEN, L. W.; UYUP, M. K. A. Effect of treatment on water absorption behavior of natural fiber 


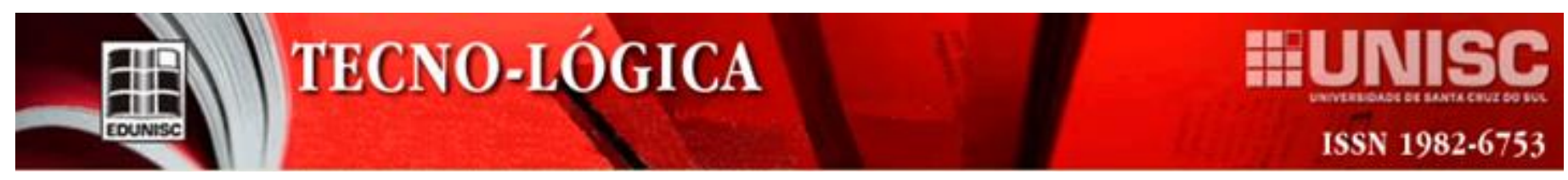

- reinforced polymer composites. Mechanical and Physical Testing of Biocomposites, Fibre-Reinforced Composites and Hybrid Composites. Woodhead Publishing Series in Composites Science and Engineering. p. 141-156, 2019.

[35] MULINARI, D. R. Comportamento Térmico, Mecânico e Morfológico dos Compósitos de Polietileno de Alta Densidade Reforçados com Fibras de Celulose do Bagaço de Cana de Açúcar. 2009. 112f. Tese (Doutorado em Engenharia Mecânica na Área de Materiais) - Faculdade de Engenharia, Universidade Estadual Paulista, UNESP, Guaratinguetá/SP.

[36] CORRADINI, E.; ROSA, M. F.; MACEDO, B. P.; PALADINI, P. D.; MATTOSO, L .H. C.Composição Química, Propriedades Mecânicas e Térmicas da Fibra de Frutos de Cultivares de Coco Verde. Revista Brasileira Frutífera. vol. 31, p.837-846, 2009.

[37] BRITO, A. D. Caracterização Química de Resíduo de Café (Borra) e Seu Efeito em Cafeeiro Orgânico Adensado e em Plântulas Decafeeiro e Tomateiro. 2013. 141f. Tese (Doutorado em Agronomia) - Universidade de Brasília, Brasília.

[38] Rodolfo Cardoso de Jesus. Processamento e Caracterização de Biocompósitos de PHB com Fibras Naturais de Coco e Sisal Acetiladas e NãoAcetiladas. 2008. 86f. Dissertação (Mestrado em Ciência e Tecnologia de Materiais) - Universidade Estadual de Campinas, Faculdade de Engenharia Química, UNICAMP, Campinas/SP.

[39] PASSADOR, F. R.; BACKES, E. H.; TRAVAIN, D. R.; FILHO, A. R.; PESSAN, L. A. Nanocompósitos de Blendas HDPE/LLDPE e OMMT - Parte I: Avaliação das Propriedades Termo-mecânicas e da Resistência ao Intemperismo. Polímeros. vol. 23, p. 521-530, 2013.

[40] Elanchezhian, C.; Ramnath, V. R.; Ramakrishnan, G.; Rajendrakumar, M.; Saravanakumar, M. K. Review on mechanical properties of natural_fiber composites. Materials Today: Proceedings. vol. 5, p. 1785-1790, 2018.

[41] BUJJIBABU, G.; DAS, V. C.; RAMAKRISHNA, M.; NAGARJUNA, K. Mechanical And Water Absorption Behavior Of Natural Fibers Reinforced Polypropylene Hybrid Composites. Materials Today: Proceedings. vol. 5, p. 12249-12256, 2018.

ORCID

Erika Bomfim dos Santos: https://orcid.org/0000-0002-5347-5275

Larissa Stieven Montagna: https://orcid.org/0000-0002-7947-3112

Fábio Roberto Passador: https://orcid.org/0000-0001-5239-5962 